\title{
A NOVEL DNA VACCINE FOR REDUCTION OF PRRSV-INDUCED NEGATIVE IMMUNOMODULATORY EFFECTS: A PROOF OF CONCEPT
}

\begin{abstract}
Sanipa Suradhat ${ }^{\mathrm{a}, \mathrm{d},{ }^{*}}$, Piya Wongyanin ${ }^{\mathrm{b}, 1}$, Sawang Kesdangsakonwut ${ }^{\mathrm{a}}$, Komkrich Teankum ${ }^{\mathrm{a}}$, Mongkol Lumyai ${ }^{\mathrm{c}}$, Sittikorn Triyarach ${ }^{\mathrm{c}, 2}$, and Roongroje Thanawongnuwech ${ }^{\mathrm{a}, \mathrm{d}}$
\end{abstract}

\footnotetext{
${ }^{a}$ Faculty of Veterinary Science, Chulalongkorn University, Pathumwan Bangkok 10330, Thailand

${ }^{b}$ Inter-department of Medical Microbiology, Graduate School, Chulalongkorn University, Pathumwan, Bangkok 10330, Thailand

c Thai-Denmark Swine Breeder Public Company Limited, Bang-na, Bangkok 10260, Thailand

${ }^{d}$ Center of Excellence in Emerging Infectious Diseases in Animals, Chulalongkorn University (CU-

EIDAs), Faculty of Veterinary Science, Chulalongkorn University, Pathumwan, Bangkok 10330,
} Thailand

* Corresponding author: $\quad$ Sanipa Suradhat, D.V.M., Ph.D.

Faculty of Veterinary Science, Chulalongkorn University, Henri-Dunant Rd., Pathumwan, Bangkok 10330, THAILAND Tel: 662-218-9583 Fax. 662-251-1656

E-mail: Sanipa.S@chula.ac.th

Present addresses

${ }^{1}$ Department of Medical Technology, Faculty of science and Technology, Bansomdejchaopraya Rajabhat University, Thonburi, Bangkok 10600, Thailand

2 Zoetis (Thailand) Ltd., Bangrak, Bangkok 10500, Thailand 
3 Background: Viral-induced interleukin (IL)-10 and regulatory T lymphocytes (Tregs) are believed to play a major role in shaping the immunological and clinical outcomes following Porcine Reproductive and Respiratory Syndrome virus (PRRSV) infection. Recently, it has been shown that PRRSV nucleocapsid $(\mathrm{N})$ protein can induce IL-10 production which is essential for induction of PRRSVspecific Tregs. We hypothesized that immunity to $\mathrm{N}$ protein should reduce PRRSV-induced negative immunomodulatory effects which will be essential for establishing proper anti-PRRSV immunity in infected pigs.

Objectives: To investigate the immunomodulatory effects of DNA vaccine encoding a linearized, truncated form of PRRSV-N protein (pORF7t) which was designed to preferentially induce cellmediated immunity against PRRSV $\mathrm{N}$ protein.

Method: Immunomodulatory effects of the novel DNA vaccine were investigated in an experimental vaccinated-challenged model. In addition, long-term immunomodulatory effects of the DNA vaccine were investigated in vaccinated pigs kept at the PRRSV-positive environment until the end of the fattening period. Pigs were vaccinated either prior to or following natural PRRSV infection.

Result: The results indicated that pORF7t could modulate the anti-PRRSV immune responses and promote the control of viral replication in the vaccinated-challenged pigs. Immunized pigs exhibited increased numbers of PRRSV-specific activated $\mathrm{CD} 4^{+} \mathrm{CD} 25^{+}$lymphocytes, reduced numbers of PRRSV-specific Tregs, and rapid viral clearance following infection. In a long-term study, regardless of the time of vaccination, DNA vaccine could modulate the host immune responses, resulted in enhanced PRRSV-specific IFN- $\gamma$ producing cells, and reduced numbers of PRRSV-specific Tregs, without evidence of enhanced antibody responses. No vaccine adverse reaction was observed throughout the study.

Conclusion: This study revealed the novel concept that PRRSV-specific immunity can be modulated by induction of cell-mediated immunity against the nucleocapsid protein. This concept could potentially benefit the development of PRRSV management and control strategies.

Keyword: PRRSV, DNA vaccine, nucleocapsid protein, Tregs 


\section{Introduction}

Infection with Porcine reproductive and respiratory syndrome virus (PRRSV) usually induces slow and weak anti-viral immune responses, leading to persistent infection and immunosuppression [1-5]. The lack of anamnestic responses observed following PRRSV exposure in previously vaccinated pigs [6, 7] implied that even the well-primed immune system was severely impaired during active PRRSV infection. Numerous reports indicate that PRRSV can evade and suppress host immune responses through several mechanisms [4, 8-11]. Among these mechanisms, inductions of interleukin-10 (IL-10) and regulatory T lymphocytes (Tregs) are believed to play a major role in shaping the immunological and clinical outcomes following PRRSV infection [3, 12-14]. Interleukin-10 is essential for induction of PRRSV-specific Tregs [15]. The role of PRRSV-specific Tregs has also been linked to delayed induction of cellular responses and increased susceptibility of bacterial infection in infected hosts $[5,16,17]$.

PRRSV nucleocapsid (N) protein, a highly conserved non-glycosylated protein, is expressed abundantly in PRRSV-infected cells [10, 18, 19]. PRRSV-N protein possesses several negative immunomodulatory properties including induction of IL-10 $[15,20]$ and inhibition of type I interferon production [21]. Induction of IL-10 by N protein was found to rely on the conformational structure of the protein $[15,20]$. In addition, induction of $\mathrm{N}$-specific antibodies may contribute to antibodydependent enhancement (ADE) of infection, by increasing viral opsonization, during the natural infection [22].

We previously hypothesized that reduction of PRRSV-induced negative immunomodulatory effects should restore proper establishment anti-PRRSV immunity and better viral control in infected pigs [9]. Vaccines which can prime the immune responses against the immunomodulatory protein should reduce PRRSV-induced negative immunomodulatory effects, and subsequently improve PRRSV-specific immunity in the infected host. For efficient immunological priming, the vaccine antigen, by itself, should not possess any immunosuppressive properties. In this study, the immunomodulatory effects of a DNA vaccine encoding a linearized, truncated form of PRRSV-N protein were investigated in an experimental pig model. The vaccine was designed to preferentially induce cell-mediated immunity against the PRRSV N protein. 


\section{Materials and methods}

\subsection{Viruses and cells}

The US genotype, Thai PRRSV strain 01NP1 (passage no. 15), isolated from PRRSVinfected pigs [23] was provided by the Chulalongkorn University-Veterinary Diagnostic Laboratory (CU-VDL). The virus was cultured and titrated in MARC-145 cells as previously described [24], and stored at $-80^{\circ} \mathrm{C}$ until needed.

\subsection{Plasmids}

The genes encoding PRRSV-N protein from the Thai PRRSV, 01NP1 strain (ORF7) or truncated PRRSV N protein (ORF7t) were subcloned into pMASIA expression vector (a gift from Dr. S. van Drunen Little-van den Hurk, Vaccine and Infectious Diseases Organization, University of Saskatchewan, Canada). The plasmids were referred to pORF7 or pORF7t, respectively. Details of cloning procedure, in vitro characterization, and plasmid amplification were described in the earlier report [15].

\subsection{Antibody and secondary conjugates}

Treg staining system: The direct conjugated monoclonal antibody (mAb), anti-swine CD25-PE (PGBL25A, IgG1), was a gift from Dr. J. A. Roth (lowa State University, Ames, IA, USA). Anti-swine CD4-FITC (74-12-4, IgG2b) conjugate were purchased from BD Biosciences (San Diego, CA, USA). Biotinylated anti-human Foxp3 mAb (236A/E7, IgG1) was purchased from eBioscience (San Diego, CA, USA). Streptavidin-PECy5 conjugate were purchased from Invitrogen (Carlsbad, CA, USA). IFN- $\gamma$ and IL-10 staining system: Anti-swine CD4-FITC (74-12-4, IgG2b), anti-swine CD8-PE (76-2-11, IgG2b), and biotinylated anti-swine IFN- $\gamma$ mAb (P2C11, IgG2a) conjugates were purchased from BD Biosciences. Anti-swine IL-10 mAb (945A4C437B1, IgG1) was purchased from Biosource (Camarillo, CA, USA). Streptavidin-PETR, goat anti-mouse IgG1-Alexaflur 647 and IgG1 isotype control were purchased from Invitrogen.

\subsection{Animal experiment}

All animal studies were conducted under the approval of Chulalongkorn University Animal Care and Use Committee, Chulalongkorn University (Animal Use Protocol No. 0731017). 
2.4.1 Experiment 1 Four-week-old, PRRSV-seronegative, crossbred pigs (4-6 pigs/group) were immunized with $500 \mu \mathrm{g}$ of pORF7t or pORF7 diluted in $200 \mu \mathrm{l} \mathrm{Ca2}{ }^{+}, \mathrm{Mg}^{+}$-free PBS (referred as PBSA), twice at 4 weeks interval (d0, d28). The plasmid was intradermally injected into both ear pinna (2 spots of $50 \mu / /$ side), using a tuberculin syringe. The control groups receiving the same amount of PBSA or null plasmid (pMASIA) were included in the study. Four weeks following the second vaccination (d56), the pigs were intranasally challenged with $5 \mathrm{ml}(2.5 \mathrm{ml} /$ nostril) of the virulent PRRSV (01NP1) at the concentration of $10^{5.5} \mathrm{TCID}_{50} / \mathrm{ml}$. The immunological parameters and clinical signs were monitored every 2 weeks and at 0,5 , and 10 days post infection (dpi). The pigs were sacrificed at $10 \mathrm{dpi}$ and subjected to virological and pathological studies. The animals were kept at the farm animal facility, Faculty of Veterinary Science, Chulalongkorn University, Nakorn Pathom campus throughout the experiment.

2.4.2 Experiment 2 The study was conducted in a PRRSV-positive farm with known PRRSV serological status located in central Thailand, approximately $160 \mathrm{~km}$ from Bangkok. The piglet production site carried approximately 3,000 sows, with continuous flow rearing system. Generally, the pigs were weaned at 4 weeks old, and kept in a nursery unit until 11 weeks old. Subsequently, the pigs were moved to a finisher unit, situated approximately $40 \mathrm{~km}$ from the nursery unit. The pigs were kept at finisher until 26-28 weeks old.

Six weeks old, male weanling pigs were randomly grouped into 5 groups (30 pigs/group). For

DNA priming (DNA-P) experiment, the pigs were intradermally immunized with $500 \mu \mathrm{g}$ of pORF7t at 6 wks old (d0), using similar protocol as in Experiment 1. For DNA treatment (DNA-T) experiment, experimental pigs were immunized with $500 \mu \mathrm{g}$ of pORF7t at the time of moving (wk 11, d35). The control groups included the groups immunized with PBSA or null plasmid (pMASIA). immunological and serological studies. At 8 days after moving ( $8 \mathrm{dpm}, \mathrm{d} 43)$, some of the pigs (6/group) were euthanized and subjected for virological and pathological studies. 
118 Experiment 1 was determined by RT-PCR using the ORF1-specific primer sets described previously 119 [23]. In Experiment 2, quantitative RT-PCR for confirmation of the presence of PRRSV-type 2 was conducted using the previously described protocol [25]. PRRSV-specific antibody responses were determined using the commercial ELISA test kit (HerdChek PRRS, IDEXX, Germany), according to the manufacturer's protocol.

\subsection{Isolation of peripheral blood mononuclear cells (PBMC) and flow cytometry}

The protocols used for isolation of porcine PBMC, was previously described in [26]. For in vitro activation, $1 \mathrm{ml}$ of PBMC $\left(6 \times 10^{6} \mathrm{cells} / \mathrm{ml}\right)$ were cultured in a well of 24 -well-plate with 0.1 multiplicity of infection (m.o.i.) of the virulent US-PRRSV (strain 01NP1), or mock infected MARC-145 lysate for $48 \mathrm{hrs}$ prior to harvesting for fluorescent staining and flow cytometric analyses. For analysis of intracellular molecules, protein transport inhibitor; monensin (GolgiStopTM, BD Biocsiences) was added to the cell culture at the $36^{\text {th }}$ hour of the incubation period. Following the 48 hours in vitro stimulation, the cells were harvested and washed and resuspended in PBSA supplemented with $0.5 \%$ BSA and $0.1 \%$ sodium azide referred as the FACS buffer. The PBMC were distributed into a roundbottomed, 96-well plate at the approximate concentration of $2 \times 10^{6}$ cells/well, and subjected to immunoflurescent staining. All staining reactions were performed at a final volume of $50 \mu \mathrm{l} / \mathrm{well}$, for 45 minutes at $4{ }^{\circ} \mathrm{C}$. Washing between each staining step and after permeabilization was performed 3 times using $200 \mu \mathrm{l}$ of FACS buffer. At the final stage, the pellet was resuspended in $200 \mu \mathrm{l}$ in $2 \%$ formaldehyde in PBSA in a 96-well plate, protected from the light, at $4{ }^{\circ} \mathrm{C}$ until analysed. All flow cytometric analyses were performed using The FC 500 MPL System (Beckman Coulter, CA, USA). [26]. Briefly, the CD4 and CD25 surface molecules were stained with 1:50 of anti-CD4-FITC and 1:25 of anti-CD25-PE antibodies. The cells were then permeabilized and fixed with a $100 \mu \mathrm{l} / \mathrm{well}$ of $50 \%$ Reagent A (Leucoperm, AbD Serotec), diluted in FACS buffer, at room temperature in the dark for 15 minute. Biotinylated anti-human Foxp3 mAb diluted 1:50 in Reagent B (Leucoperm, AbD Serotec) was added. Subsequently, 1:100 of secondary conjugate, Streptavidin-PECy5, diluted in FACS buffer was added. For intracellular cytokine staining system, the cells were stained by addition of 1:50 of anti-swine CD4-FITC and anti-swine CD8-PE mAbs. The cells were then permeabilized and fixed. 
147 Anti-swine IFN- $\gamma$ and anti-swine IL-10 mAbs diluted 1:50 in Reagent B (Leucoperm, AbD Serotec)

148 were added. Subsequently, 1:100 of secondary conjugate, Streptavidin-PETR and goat-anti mouse

149 IgG1-Alexaflur647, diluted in FACS buffer were added. The cells stained with the isotype control

150 antibody (biotinylated IgG1 antibody) were included, and used as the background cut-off. The

151 fluorescent minus one (FMO) staining controls were performed during the establishment and

152 validation of an assay.

153 The data represents mean percentage $( \pm S E M)$ of the cytokine-producing cells from the pigs in

154 the same group, which was calculated from \% cytokine producing cells obtained from the PRRSV-

155 cultured PBMC - \% cytokine producing cells obtained from the mock-cultured PBMC.

156

$157 \quad 2.8$ Statistical analyses

158 Data was analysed using analysis of variance (ANOVA) followed by Tukey's multiple

159 comparison tests. All statistical analyses were performed using GraphPad Prism for Windows

160 (GraphPad Software Incorporated, San Diego, CA, USA).

161 
3.1 The immunomodulatory effects of the PRRSV DNA vaccine in a vaccinated-challenged model

No clinical signs or vaccine-adverse reactions were observed following DNA immunization.

At $10 \mathrm{dpi}$, the presence of PRRSV in the lung tissues was evident in all challenged groups, except the group immunized with pORF7t (Table 1). Pathological findings in the respiratory tracts of the experimental pigs were summarized in Table 1. Seroconversion was only observed in the group that received pORF7 on the challenge day (1 pig), and at 10dpi (3 pigs). As nucleocapsid protein is the major epitope recognized by the anti-PRRSV antibody determined by IDEXX HerdChek ELISA assay [27], the result suggested that pORF7 immunization could prime of anti-PRRSV antibody respons in some pigs (Table 2).

Following the challenge, increased numbers of PRRSV-specific IFN- $\gamma$ and IL-10 producing cells were observed in all groups (Fig 1a, b). However, statistical differences in the numbers of cytokine producing cells among the groups were not observed due to high variability within the same group. The pigs immunized with pORF7t exhibited higher numbers of the PRRSV-specific activated Th lymphocytes (CD4 ${ }^{+} \mathrm{C} 25^{+}$cells) at d28 ( $\left.p<0.05\right)$, (Fig. 1c). The numbers of PRRSV-specific $\mathrm{CD}^{+} \mathrm{CD} 25^{+} \mathrm{Foxp}^{+}$Tregs in the group receiving the pORF7t and pORF7 were lower than those in the control groups $(p<0.05)$ at d28 (Fig. 1d).

The data indicated that the pORF7t could modulate the immune responses against PRRSV, by reduction of the viral-specific Tregs and enhance the numbers of viral-specific responder Th cells without priming or induction of $\mathrm{N}$-specific antibody responses.

3.2 The long-term study on immunomodulatory effects of the PRRSV DNA vaccine in pigs kept at a commercial, PRRSV-positive, farm

In order to investigate the long-term immunomodulatory effects of the novel DNA vaccine in immunized pigs, Experiment 2 was conducted in a PRRSV-positive farm (see materials and methods). In addition, pigs immunized either prior to (priming effect), or following PRRSV natural exposure (treatment effect) were included in the study. Six weeks old, male weanling pigs were immunized at 6 (priming, d0) or 11 (treatment, d35) weeks old, moved to the finisher unit at 11 weeks old and kept there until 26-28 weeks old. At 8 days (d43) following moving into the finisher unit, the presence of the US genotype PRRSV in the serum and lung samples was confirmed of the all 
experimental groups by quantitative RT-PCR. However, it was impossible to compare the level of viral loads among the groups due to undefined time and dose of infection within the farm setting. The pathological studies of the respiratory tracts at $\mathrm{d} 43$ revealed viral infection with secondary complication, with comparable means lung scores in all groups (data not shown).

Cellular responses-Priming experiment: Following vaccination, the pigs receiving pORF7t exhibited increased numbers of PRRSV-specific IFN- $\gamma^{+}$lymphocytes which remained higher than other groups until d70 (Fig. 2a). Following moving to the finisher unit, all groups exhibited increased numbers of PRRSV-specific IL-10 ${ }^{+}$cells and then declined after d56. The groups receiving either pORF7t or the null plasmid had significantly lower number of PRRSV-specific IL- $10^{+}$cells than the control PBSA at $\mathrm{d} 70(p<0.05)$. Only the group immunized with pORF7t exhibited gradual decrease of the PRRSVspecific IL-10 $10^{+}$cells through the end of the observation period. The pigs receiving pORF7t exhibited lower numbers of the PRRSV-specific Tregs than the plasmid control group $(p<0.05)$. After moving (d43), the numbers of PRRSV-specific Tregs increased in every group. The number of the PRRSVspecific Tregs in the pORF7t vaccinated group remained lower than the other groups throughout the experiment (Fig. 2c). The result from this experiment indicated that the DNA vaccine could modulate the anti-PRRSV cellular immune responses in the vaccinated pigs, by significantly enhancing the production of viral-specific IFN- $\gamma$, and altered the pattern of IL-10 and Tregs production following the viral exposure.

\section{Cellular responses-Treatment experiment: Immunization with pORF7t resulted in an enhanced} induction of PRRSV-specific IFN- $\gamma^{+}$cells by d43. The numbers of the PRRSV-specific IFN- $\gamma$ cells in the pORF7t group remained higher than the other groups through the end of the experiments (Fig. 2d). All pigs exhibited increased PRRSV-specific IL- $10^{+}$cells in the PBMC following moving into the finisher unit (d43). The pigs receiving pORF7t exhibited faster reduction of the PRRSV-specific IL-10 cells and had lower levels of PRRSV-specific IL-10 ${ }^{+}$cells than the other control groups until the end of the observation period (Fig. 2e). Furthermore, the pigs received pORF7t exhibited significantly lower numbers of PRRSV-specific CD4 ${ }^{+} \mathrm{CD} 25^{+}$Foxp $3^{+}$cells than the control groups throughout the experiment (Fig. 2f). The results suggested that pORF7t, even immunized concurrently with natural 
221 exposure, could still modulate the PRRSV-specific immune responses that should be benefit to the 222 host.

224 The effect of DNA immunization on anti-PRRSV antibody responses

225 Some of the pigs contained maternal-derived PRRSV-specific antibody in their serum at the 226 beginning of the experiment. The level of MDA gradually reduced until 8 wks old (d14). However, 80 -

$227100 \%$ of the pigs in every groups exhibited seroconversion at the time of moving (d35), suggesting 228 that the natural infection actually occurred in the nursery. There were no significant different in the 229 pattern of antibody responses, measured by ELISA, among the experimental groups (Table 3), 230 indicating that the vaccine had no effect on PRRSV-specific humoral immune responses. 
DNA immunization is a powerful tool for priming of PRRSV-specific cellular responses and modulating the PRRSV-induced immunomodulatory effects. Regardless of the time of immunization, single immunization of plasmid encoding ORF7t could provide long-lasting positive effects on PRRSVspecific cellular responses. Our findings are consistent with the earlier findings on the role of ORF7 gene in induction of PRRSV-specific cellular immunity [28-30]. It has been previously shown that inhibition of IL-10 production can enhance pro-inflammatory cytokine production following PRRSV infections [31, 32], and reduce PRRSV-specific Tregs [15]. Thus, the underlined mechanism of our findings is likely related to induction of $\mathrm{N}$-specific cellular responses which resulted in reduction of Ninduced immunomodulatory activities, i.e. IL-10 production, during the infection period. The enhanced PRRSV-specific IFN- $\gamma$ producing cells and reduced numbers of Tregs in the vaccinated group should enhance induction of anti-viral immunity and host immune status, resulted in improvement in the clinical outcomes following PRRSV exposure. One limitation of the long-term study was that we were not able to assess reduction in pneumonia and clinical score in the vaccinated pigs at the end of finishing stage. The reason for this was due to an unexpected problem on animal feed at the finisher site, resulted in poor nutritional and health status of the experimental pigs. This prohibited us to perform assessment on the clinical score and feed efficiency at the end of the trial.

It should be noted that the immunomodulatory effects of the plasmid encoding a full-length ORF7 (pORF7) and linearized ORF7 (pORF7t) are different. The pORF7t induced better positive immunomodulatory effects and viral clearance than that of pORF7. Earlier studies indicate that the ability to upregulate IL-10 gene by PRRSV N protein relies on the conformational structure and is significantly reduced in the truncated form $[15,20]$. Therefore, the difference in this finding may partly be explained through the different immunomodulatory properties of the expressed protein during the priming phase. In addition, it has been previously shown that PRRSV N protein strongly inhibits IFN- $\beta$ production [21]. It is currently unclear if the protein conformational is essential for other immunomodulatory activities of the protein. Further investigation will be needed to confirm this notion. As pORF7t did not induced anti-PRRSV antibodies, this property would be more preferable for minimizing the induction of $A D E$ and for routine seromonitoring. should improve the anti-viral immune responses during PRRSV infection, rather than direct induction 
of PRRSV-protective immunity in vaccinated pigs. The positive immunomodulatory property of pORF7t implies the possibility for enhancing the efficacy of the currently available vaccines. Presently, it is well accepted that modified live vaccines (MLV) provide the best protection among the commercially available vaccines. However, MLV-induced immunity is usually delayed and does not provide complete protection against heterologous viruses [8,33]. Similar to the virulent PRRSV strains, MLV also induces Tregs which affect the immunocompetency of the host [17]. It is envisaged that priming with DNA vaccine should reduce MLV-induced immunomodulatory activities (if any) and, subsequently, improve vaccine-induced protective immunity. As $\mathrm{N}$ protein are highly conserved among the PRRSV strains and genotypes [30], the vaccine should effectively modulate the immune responses against the heterologous PRRSV strains as well. By selecting appropriate MLV booster, the heterologous, DNA-MLV, prime-boost regimen should provide a long-term benefit in the vaccinated pigs. This strategy could provide more advantage in heavily infected areas or in combating with the highly virulent PRRSV strains. The notion is currently under investigated.

In summary, our study revealed the novel concept to modulate PRRSV-specific immunity by induction of cell-mediated immunity against the nucleocapsid protein. The prototype vaccine could potentially benefit the PRRSV management and control. This concept may also be useful for other pathogen with similar immunomodulatory activities.

\section{Acknowledgements}

The authors are grateful to Dr. S. van Drunen Little-van den Hurk, Vaccine and Infectious Diseases Organization, University of Saskatchewan, Canada, Prof. James A. Roth and Dr. Ratree Platt (lowa State University, USA) for their kind provision of the plasmid and monoclonal antibodies used in this study. The authors wish to specially thank Thai-Denmark Swine Breeder Public Co. Ltd. and their staffs for providing the animal facility, and technical assistance during the long-term study. We thank Dr. Pravina Kitikoon, the staff of Veterinary Diagnostic Laboratories and the graduate students in Veterinary Pathobiology program, Faculty of Veterinary Science, Chulalongkorn University for their technical assistance. This work is supported by The Thailand Research Fund, Office of the Higher Education Commission, Chulalongkorn University, and The Royal Golden Jubilee Ph.D. Program (P.W). 


\section{Legends}

293 Figure 1 Numbers of PRRSV-specific IFN- $\gamma^{+}$(a) and IL-10 ${ }^{+}$cells (b), $\mathrm{CD}^{+}{ }^{+} \mathrm{CD} 25^{+}$(c) in the PBMC and $294 \mathrm{FoxP}^{+} \mathrm{CD}^{+} \mathrm{CD}_{25} 5^{+}$(d) from the pigs in Experiment 1. Pigs were immunized with the vaccine indicated 295 in the legend on d0 and d28, and received PRRSV inoculation on d56. ${ }^{a}$ indicates differences

$296(p<0.05)$ from the other treatments. ${ }^{b}$ indicates differences $(p<0.05)$ between the groups receiving the 297 DNA vaccine (pORF7t or pORF7) and the controls (null or PBSA).

298 Figure 2 The numbers of PRRSV-specific IFN- $\gamma^{+}$cells, IL-10+ cells in the PBMC, and the 299 Foxp $3^{+} \mathrm{CD} 4^{+} \mathrm{CD} 25^{+}$subpopulations from the priming $(\mathrm{a}, \mathrm{b}, \mathrm{c})$ and treatment $(\mathrm{d}, \mathrm{e}, \mathrm{f})$ experiment. Pigs 300 were vaccinated with pORF7t (solid square), null plasmid (triangle), or PBSA (diamond) on d0 or d35, 301 and moved to the finisher at d35. The freshly isolated porcine PBMC samples were cultured with 0.1 302 m.o.i. of US-PRRSV (strain 01NP1), or mock-infected MARC-145 lysate for $48 \mathrm{hrs}$ prior to fluorescent

303 staining and flow cytometric analyses. ${ }^{a}$ indicates statistical difference from other groups, at $p<0.05 .{ }^{b}$ 304 indicates statistical difference between the pORF7t and null plasmid, at $p<0.05 .{ }^{\circ}$ indicates statistical 305 different between the pORF7t and PBSA, at $p<0.05 .{ }^{d}$ indicates statistical different between the null 306 and PBSA, at $p<0.05)$. 
Table 1

Table 1 Virological and pathological findings at $10 \mathrm{dpi}$ (Experiment 1)

\begin{tabular}{lllll}
\hline & \multicolumn{4}{l}{ Experimental group (n) } \\
\cline { 2 - 5 } & PBSA (4) & Null (6) & pORF7 (6) & pORF7t (6) \\
\hline \% Positive lung samples (+ve/n) & $50(2 / 4)$ & $50(3 / 6)$ & $33.3(2 / 3)$ & $0(0 / 6)$ \\
\hline \% Pathological lesions at the lungs & & & & \\
Pneumonia & 75.0 & 33.2 & 33.2 & 50.0 \\
Lymphadenopathy & 50.0 & 16.6 & 0 & 16.6 \\
(Tracheobroncheal Ln.) & 0 & 16.6 & 16.6 & 0 \\
Fibrinous pleuritis & 0 & &
\end{tabular}


Table 2

Table 2 Anti-PRRSV ELISA antibody responses in the experimental pigs during Experiment 1

\begin{tabular}{lllll}
\hline \multirow{2}{*}{ Group } & \multicolumn{4}{l}{ Mean S/P ratio (\% seroconversion $\left.{ }^{\mathrm{a}}\right)$} \\
\cline { 2 - 5 } & $\mathbf{d} \mathbf{0}$ & $\mathbf{d 5 6}(\mathbf{0} \mathrm{dpi})$ & $\mathbf{d 6 1}(\mathbf{5 ~ d p i )}$ & $\mathbf{d 6 6}(\mathbf{1 0} \mathrm{dpi})$ \\
\hline ORF7t & $0.08(0)$ & $0.02(0)$ & $0.02(0)$ & $0.03(0)$ \\
ORF7 & $0.07(0)$ & $\mathbf{0 . 1 5}(\mathbf{1 6 . 6 )}$ & $\mathbf{0 . 0 4}(\mathbf{0})$ & $\mathbf{0 . 4 6}(\mathbf{5 0})$ \\
Null & $0.11(0)$ & $0.04(0)$ & $0.01(0)$ & $0.02(0)$ \\
PBSA & $0.06(0)$ & $0.03(0)$ & $0.02(0)$ & $0.01(0)$
\end{tabular}

${ }^{a} \mathrm{~S} / \mathrm{P}$ ratio $\geq 0.4=$ positive 
Table 3

Table 3 Anti-PRRSV ELISA antibody responses in the experimental pigs during Experiment 2

\begin{tabular}{lllllllll}
\hline \multirow{2}{*}{ Group } & \multicolumn{7}{c}{ Mean S/P ratio ${ }^{\text {a }}$ \% seropositive pigs ${ }^{\mathrm{b}}$ ) } \\
\cline { 2 - 8 } & $\mathrm{d} 0$ & $\mathrm{~d} 14$ & $\mathrm{~d} 35$ & $\mathrm{~d} 43$ & $\mathrm{~d} 56$ & $\mathrm{~d} 70$ & $\mathrm{~d} 82$ & $\mathrm{~d} 112$ \\
\hline PBSA & $0.25(33.33)$ & $0.21(33.33)$ & $1.31(100)$ & $2.28(100)$ & $1.99(80)$ & $2.29(100)$ & $1.42(100)$ & $1.36(100)$ \\
pORF7t-P & $0.17(16.67)$ & $0.09(0)$ & $1.24(100)$ & $1.35(100)$ & $1.15(100)$ & $1.26(80)$ & $1.02(100)$ & $1.256(100)$ \\
Null-P & $0.30(33.33)$ & $0.239(0)$ & $1.22(100)$ & $0.926(100)$ & $2.16(100)$ & $1.36(100)$ & $1.01(80)$ & $1.00(60)$ \\
pORF7t-T & nd & nd & $1.19(80)$ & $1.29(100)$ & $1.16(100)$ & $1.09(100)$ & $1.65(100)$ & $1.29(100)$ \\
Null-T & nd & nd & $0.96(80)$ & $1.07(80)$ & $0.98(80)$ & $1.72(80)$ & $0.83(100)$ & $1.21(100)$
\end{tabular}

${ }^{a}$ mean OD values from 5 pigs/group

${ }^{b} \mathrm{~S} / \mathrm{P}$ ratio $\geq 0.4$ = seropositive 
[1] Van Reeth K, Nauwynck H. Proinflammatory cytokines and viral respiratory disease in pigs. Vet Res. 2000;31:187-213.

[2] Thanawongnuwech R, Brown GB, Halbur PG, Roth JA, Royer RL, Thacker BJ. Pathogenesis of porcine reproductive and respiratory syndrome virus-induced increase in susceptibility to Streptococcus suis infection. Vet Pathol. 2000;37:143-52.

[3] Lopez OJ, Osorio FA. Role of neutralizing antibodies in PRRSV protective immunity. Vet Immunol Immunopathol. 2004;102:155-63.

[4] Mateu E, Diaz I. The challenge of PRRS immunology. Vet J. 2008;117:345-51.

[5] Cecere TE, Todd SM, Leroith T. Regulatory T cells in arterivirus and coronavirus infections: do they protect against disease or enhance it? Viruses. 2012;4:833-46.

[6] Zuckermann FA, Garcia EA, Luque ID, Christopher-Hennings J, Doster A, Brito M, et al. Assessment of the efficacy of commercial porcine reproductive and respiratory syndrome virus (PRRSV) vaccines based on measurement of serologic response, frequency of gamma-IFNproducing cells and virological parameters of protection upon challenge. Vet Microbiol. 2007;123:69-85.

[7] Martelli P, Gozio S, Ferrari L, Rosina S, De Angelis E, Quintavalla C, et al. Efficacy of a modified live porcine reproductive and respiratory syndrome virus (PRRSV) vaccine in pigs naturally exposed to a heterologous European (Italian cluster) field strain: Clinical protection and cellmediated immunity. Vaccine. 2009;27:3788-99.

[8] Yoo D, Song C, Sun Y, Du Y, Kim O, Liu HC. Modulation of host cell responses and evasion strategies for porcine reproductive and respiratory syndrome virus. Virus Res. 2010;154:48-60.

[9] Thanawongnuwech R, Suradhat S. Taming PRRSV: Revisiting the control strategies and vaccine design. Virus Res. 2010;154:133-40.

[10] Snijder EJ, Kikkert M, Fang Y. Arterivirus molecular biology and pathogenesis. J Gen Virol. 2013;94:2141-63.

[11] Huang C, Zhang Q, Feng WH. Regulation and evasion of antiviral immune responses by porcine reproductive and respiratory syndrome virus. Virus Res. 2014.

[12] Meng XJ. Heterogeneity of porcine reproductive and respiratory syndrome virus: implications for current vaccine efficiency and future vaccine development. Vet Microbiol. 2000;74:309-29.

[13] Meier WA, Galeota J, Osorio FA, Husmann RJ, Schnitzlein WM, Zuckermann FA. Gradual development of the interferon-gamma response of swine to porcine reproductive and respiratory syndrome virus infection or vaccination. Virology. 2003;309:18-31.

[14] Suradhat S, Kesdangsakonwut S, Sada W, Buranapraditkun S, Wongsawang S, Thanawongnuwech $R$. Negative impact of porcine reproductive and respiratory syndrome virus infection on the efficacy of classical swine fever vaccine. Vaccine. 2006;24:2634-42.

[15] Wongyanin P, Buranapraditkul S, Yoo D, Thanawongnuwech R, Roth JA, Suradhat S. Role of porcine reproductive and respiratory syndrome virus nucleocapsid protein in induction of interleukin-10 and regulatory T-lymphocytes (Treg). J Gen Virol. 2012;93:1236-46.

[16] Silva-Campa E, Flores-Mendoza L, Resendiz M, Pinelli-Saavedra A, Mata-Haro V, Mwangi W, et al. Induction of $T$ helper 3 regulatory cells by dendritic cells infected with porcine reproductive and respiratory syndrome virus. Virology. 2009;387:373-9.

[17] LeRoith T, Hammond S, Todd SM, Ni Y, Cecere T, Pelzer KD. A modified live PRRSV vaccine and the pathogenic parent strain induce regulatory $T$ cells in pigs naturally infected with Mycoplasma hyopneumoniae. Vet Immunol Immunopathol. 2011;140:312-6.

[18] Nelson EA, Christopher-Hennings J, Benfield DA. Serum immune responses to the proteins of porcine reproductive and respiratory syndrome (PRRS) virus. J Vet Diagn Invest. 1994;6:410-5.

[19] Dea S, Gagnon CA, Mardassi H, Pirzadeh B, Rogan D. Current knowledge on the structural proteins of porcine reproductive and respiratory syndrome (PRRS) virus: comparison of the North American and European isolates. Arch Virol. 2000;145:659-88.

[20] Liu X, Fan B, Bai J, Wang H, Li Y, Jiang P. The N-N non-covalent domain of the nucleocapsid protein of type 2 porcine reproductive and respiratory syndrome virus enhances induction of IL10 expression. J Gen Virol. 2015. doi: 10.1099/vir.0.000061. [Epub ahead of print]

[21] Sagong M, Lee C. Porcine reproductive and respiratory syndrome virus nucleocapsid protein modulates interferon-beta production by inhibiting IRF3 activation in immortalized porcine alveolar macrophages. Arch Virol. 2011;156:2187-95.

[22] Cancel-Tirado SM, Evans RB, Yoon KJ. Monoclonal antibody analysis of porcine reproductive and respiratory syndrome virus epitopes associated with antibody-dependent enhancement and neutralization of virus infection. Vet Immunol Immunopathol. 2004;102:249-62. 
[23] Thanawongnuwech R, Amonsin A, Tasanakit A, Damrongwatanapokin S. Genetics and geographical variation of porcine reproductive and respiratory syndrome virus (PRRSV) in Thailand. Vet Microbiol. 2004;101:9-21.

[24] Thanawongnuwech R, Halbur PG, Ackermann MR, Thacker EL, Royer RL. Effects of low (modified-live virus vaccine) and high (VR-2385) virulence strains of porcine reproductive and respiratory syndrome virus (PRRSV) on pulmonary clearance of copper particles in pigs. Veterinary Pathology. 1998;35:398-406.

[25] Egli C, Thur B, Liu L, Hofmann MA. Quantitative TaqMan RT-PCR for the detection and differentiation of European and North American strains of porcine reproductive and respiratory syndrome virus. J Virol Methods. 2001;98:63-75.

[26] Wongyanin P, Buranapraditkun S, Chokeshai-Usaha K, Thanawonguwech R, Suradhat S. Induction of inducible $\mathrm{CD} 4^{+} \mathrm{CD} 25^{+} \mathrm{Foxp} 3^{+}$regulatory $\mathrm{T}$ lymphocytes by porcine reproductive and respiratory syndrome virus (PRRSV). Vet Immunol Immunopathol. 2010;133:170-82.

[27] Plagemann PG. Peptide ELISA for measuring antibodies to N-protein of porcine reproductive and respiratory syndrome virus. J Virol Methods. 2006;134:99-118.

[28] Xue Q, Zhao YG, Zhou YJ, Qiu HJ, Wang YF, Wu DL, et al. Immune responses of swine following DNA immunization with plasmids encoding porcine reproductive and respiratory syndrome virus ORFs 5 and 7, and porcine IL-2 and IFNgamma. Vet Immunol Immunopathol. 2004;102:291-8.

[29] Rompato G, Ling E, Chen Z, Van Kruiningen H, Garmendia AE. Positive inductive effect of IL-2 on virus-specific cellular responses elicited by a PRRSV-ORF7 DNA vaccine in swine. Vet Immunol Immunopathol. 2006;109:151-60.

[30] Diaz I, Pujols J, Ganges L, Gimeno M, Darwich L, Domingo M, et al. In silico prediction and ex vivo evaluation of potential T-cell epitopes in glycoproteins 4 and 5 and nucleocapsid protein of genotype-I (European) of porcine reproductive and respiratory syndrome virus. Vaccine. 2009;27:5603-11.

[31] Charerntantanakul W, Kasinrerk W. Interleukin-10 antisense oligodeoxynucleotide suppresses IL-10 expression and effects on proinflammatory cytokine responses to porcine reproductive and respiratory syndrome virus. Viral Immunol. 2010;23:425-35.

[32] Charerntantanakul W, Kasinrerk W. Plasmids expressing interleukin-10 short hairpin RNA mediate IL-10 knockdown and enhance tumor necrosis factor alpha and interferon gamma expressions in response to porcine reproductive and respiratory syndrome virus. Vet Immunol Immunopathol. 2012;146:159-68.

[33] Charerntantanakul W. Porcine reproductive and respiratory syndrome virus vaccines: Immunogenicity, efficacy and safety aspects. World J Virol. 2012;1:23-30. 
Figure 1

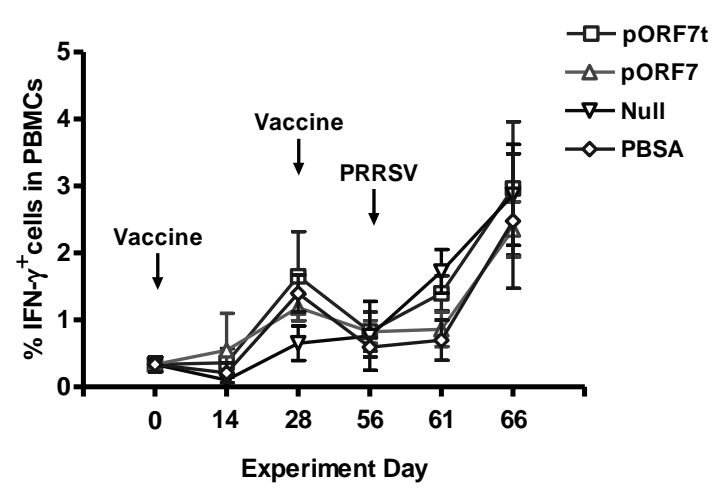

C

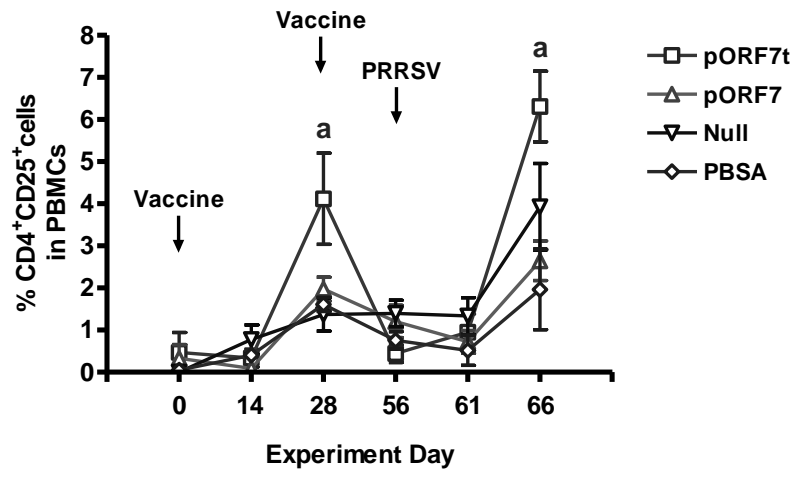

b

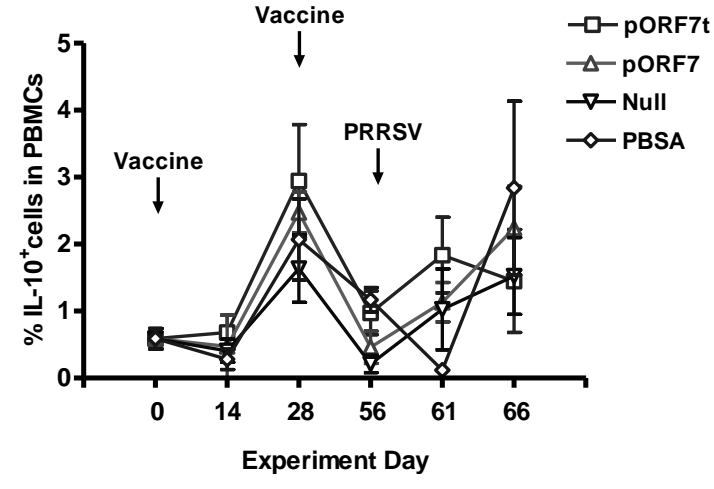

d

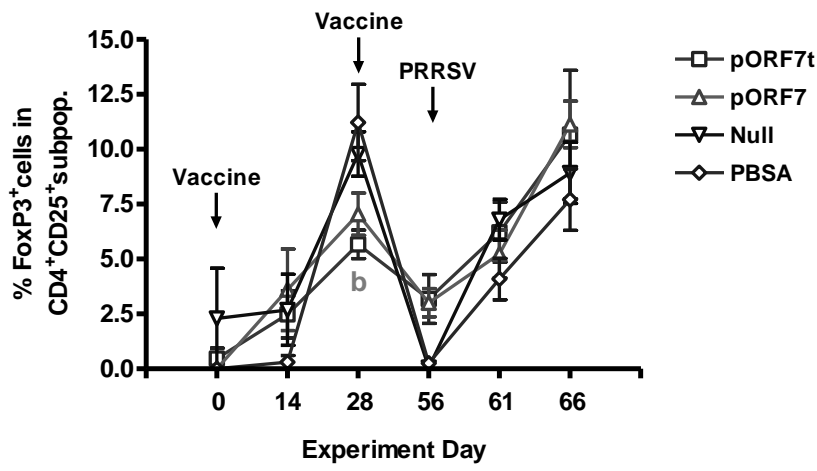


Figure 2

Experiment 2-Priming

a

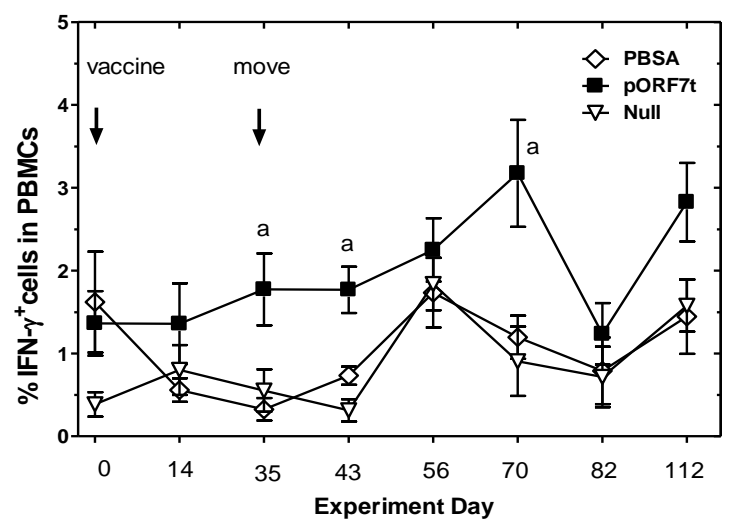

b

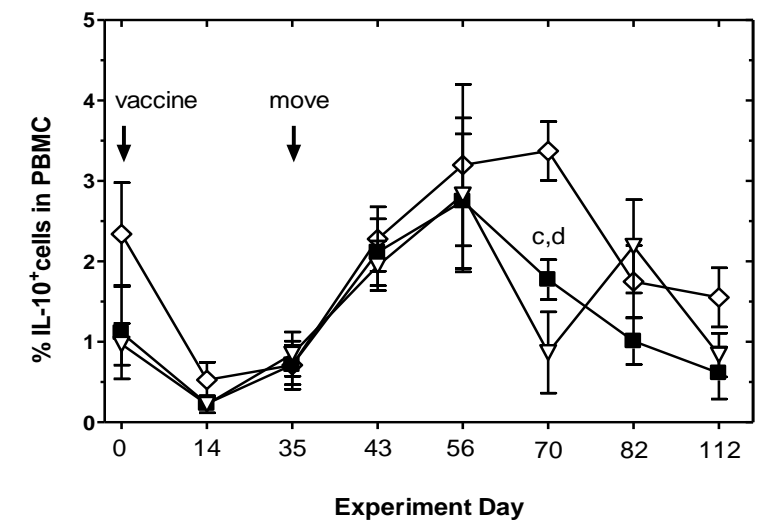

C

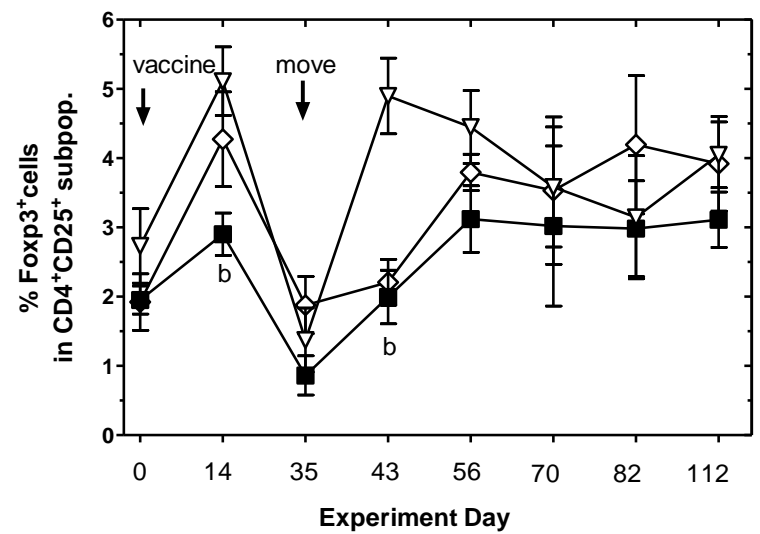

Experiment 2-Treatment

d

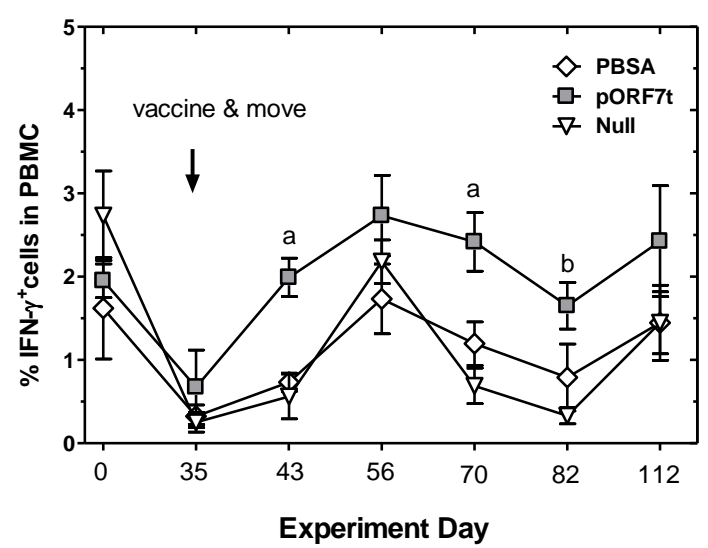

e
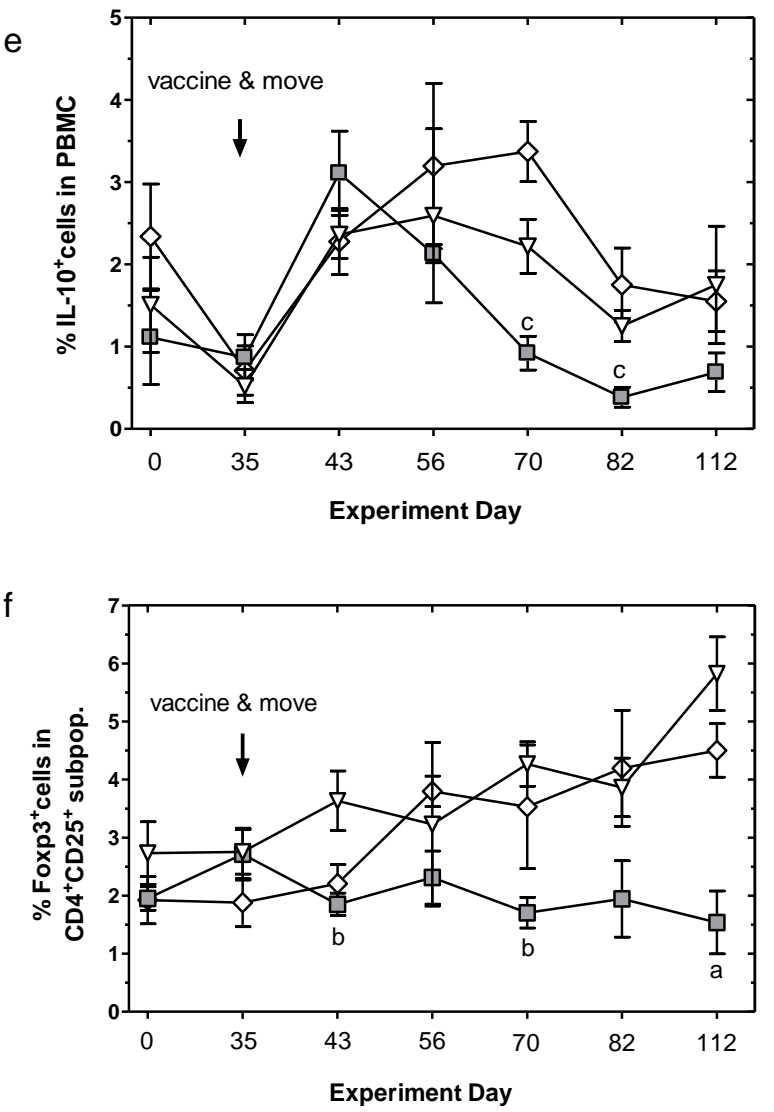